\title{
ACE 36
}

\section{ESPACIO PÚBLICO CONTEMPORÁNEO EN LA CIUDAD DEL SIGLO XXI. ¿CRISIS O TRASFORMACIÓN?}

María Teresa Pérez Bourzac

Cómo citar este artículo: PÉREZ BOURZAC, M. T. Espacio público contemporáneo en la ciudad del Siglo XXI. ¿Crisis o trasformación? [en línea] Fecha de consulta: dd-mm-aa. En: ACE: Architecture, City and Environment = Arquitectura, Ciudad y Entorno, 12 (36): 131-140, 2018. DOI: http://dx.doi.org/10.5821/ace.12.36.5300 ISSN: 1886-4805.

\section{Architecture, City, and Environment




\section{ACE 36}

Electronic offprint

Separata electrónica

\section{CONTEMPORARY PUBLIC SPACE IN THE CITY OF THE 21ST CENTURY; CRISIS OR TRANSFORMATION?}

Key words: Public space; city; shopping centers (malls)

\section{Structured abstract}

\section{Objective}

To analyze the role of shopping centers as public spaces in the contemporary city, that become, because of their appropriation, functions and meanings, in spaces that are representative of the leisure and recreation of modern life. The malls supplant the historical function of the traditional square and street to transform into places that represent the upper and middle classes' utopia and aspirations; as well as the aspirations of a part of the less favored in their ascent project towards higher levels of the social scale; and they are constituted as islands of particular functions in the city's context; unmistakable meaning of the contemporary city's fragmentation, dispersion and specialization. The public spaces and the total structure of cities, the formal and functional codes of its identity are implicated nuclearly in the modern city's process of modernizing transformation, phenomenon that allows its accomplishment under the influence of the XXI century's construction scenarios.

\section{Methodology}

Using theoretical references from authors like Borja \& Muxi (2003), Fuentes Gómez (2005) and Baudrillard (1978) and a partaker observation and analysis, these architectural structures are studied. These places are designed not to be seen and admired from the outside, but to be enjoyed inwardly; exclusiveness spaces for people that identify not as citizens, but as consumers.

\section{Conclusions}

This investigation is targeted towards specialists and professionals that carry out urban studies, and specifically analysis of these public spaces that convert in a species of autonomous nodes, if their link with the urban structure is analyzed; but if we observe their uses and meanings, they become independent spaces of civic life that contribute to the dispersion and individualized concentration that confirm the characteristics of the contemporary city.

\section{Originality}

The characterization of shopping centers (spaces specializing in leisure and consumption) as public spaces of the contemporary society leads us to confirm how the public space has positioned itself as a relevant issue in the current debates of the city. 


\section{EL ESPACIO PÚBLICO CONTEMPORÁNEO EN LA CIUDAD DEL SIGLO XXI. ¿CRISIS O TRASFORMACIÓN? ${ }^{1}$}

\section{PÉREZ BOURZAC, María Teresa ${ }^{2}$}

Remisión inicial: 25-07-2017

Aceptación: 05-12-2017

Remisión definitiva: 18-01-2018

Palabras clave: Espacio público; ciudad; centros comerciales (malls)

\section{Resumen estructurado}

\section{Objetivo}

Analizar el papel de los centros comerciales como espacios públicos de la ciudad contemporánea, que se convierten, por su apropiación, funciones y significados, en espacios públicos representativos del ocio y el esparcimiento de la vida moderna. Los 'malls' suplantan la función histórica de la plaza y la calle tradicional para transformarse en lugares que representan la utopía y aspiraciones de las clases medias y altas; como también de una parte de los sectores menos favorecidos en su proyecto de ascenso a niveles más altos de la escala social; y se constituyen como islas de funciones particulares en el contexto de la ciudad; significado inequívoco de la fragmentación, dispersión y especialización de la ciudad contemporánea. Los espacios públicos y la estructura total de las urbes, los códigos formales y funcionales de su identidad se ven implicados nuclearmente en el proceso de transformación modernizadora de la ciudad contemporánea, fenómeno que permite su realización bajo la influencia de los escenarios en construcción del siglo XXI.

\section{Metodología}

Utilizando referencias teóricas de autores como Borja \& Muxi (2003), Fuentes Gómez (2005) y Baudrillard (1978) y una observación y análisis participante, se estudian estas estructuras arquitectónicas diseñadas no para ser vistas y admiradas desde afuera, sino disfrutadas interiormente; espacios de exclusividad para personas identificadas no como ciudadanos sino como consumidores.

\section{Conclusiones}

Esta investigación está dirigida a especialistas y profesionales que realizan estudios urbanos y específicamente análisis de estos espacios públicos que se convierten en una especie de nodos autónomos; si se analiza su vinculación con la estructura urbana, pero si observamos sus usos y significados, se convierten en espacios independientes de la vida ciudadana que

\footnotetext{
1 Esta investigación forma parte de un proyecto de investigación más amplio, relativo al estudio del espacio público contemporáneo en la ciudad de Guadalajara, México.

2 Doctora en Historia del Arte, Profesor investigador titular "C" Centro Universitario de Arte, Arquitectura y Diseño (CUAAD), Universidad de Guadalajara (UdG), Jalisco, México. Miembro del Sistema Nacional de Investigadores. Correo electrónico: matepebo@gmail.com
} 
contribuyen a la dispersión y a la concentración individualizada que confirma las características de la ciudad contemporánea.

\section{Originalidad}

La caracterización de los centros comerciales (espacios especializados en el ocio y el consumo) como espacios públicos de la sociedad contemporánea nos lleva a confirmar como el espacio público se ha posicionado como un tema relevante en los debates actuales de la ciudad.

\section{Introducción. Anticipada reflexión}

Podemos afirmar, en su significado, que el espacio público es desde hace varios años un tema de actualidad y de debate en foros académicos y seminarios de investigación. La importancia de los trabajos y debates que tratan de la comprensión del tema y su análisis, especialmente en un presente que deviene de dinámicos procesos de transformación urbana, nos empuja a adentrarnos en la reflexión y el conocimiento acerca del uso de lo que se identifica hoy día el espacio público. Las razones son varias y podrían ser muy discutibles. Sin embargo, el desarrollo de los intereses inmobiliarios y de la propiedad privada como valor particular y la generación acelerada de plusvalía, que tienen como escenario la ciudad, nos ha llevado a encontrarnos en el ámbito de la transformación del espacio público en el contexto neoliberal de nuestras ciudades, en el que se desarrollan hoy, para indagar sobre las claves de asunción del espacio público, germen de la estructura de la ciudad como lugar de significado y tradición, y su paso hacia el impulso de lo privado como atractor de trasferencia de una nueva unidad espacial público-privado que es producto e inserción de la sociedad de consumo en el espacio de la ciudad. El diseño de las calles para uso casi exclusivo del automóvil privado, la pérdida de espacio público/verde en pro de las infraestructuras de traslado, el abandono de los espacios públicos tradicionales, y especialmente la oferta del comercio en contenedores especializados; que se manifiesta en la proliferación de las plazas o centros comerciales (también llamados "malls"), entre otros, son evidencia de cambios consustanciales a los que trae consigo el transcurso de la modernidad hacia una nueva fase de la historia.

Las preguntas que han surgido durante la elaboración de esta investigación, se insertan en el interés de un redescubrimiento del valor cultural de lo público en lo urbano, y su importancia en la significación simbólica y la creación de identidad a través del espacio público como lugar existencial de una comunidad determinada. Jordi Borja y Zaida Muxí, en su libro "El espacio público, ciudad y ciudadanía" (2003) apuntan diversos factores que han contribuido a la crisis de los espacios públicos en la actualidad, y señalan que: "A lo largo del siglo XX diversos factores han provocado la crisis del espacio público urbano: la dinámica de la propiedad privada, la prioridad pública y privada a los programas inmobiliarios, la ocupación exclusiva del espacio "circulatorio" por parte del automóvil, la oferta comercial cerrada, la inseguridad ciudadana...en consecuencia para ... recuperar el espacio público se ha tendido a convertirlo en un elemento especializado, un "equipamiento" más de la ciudad... que pierde así sus dos funciones fundacionales...: Dar forma y sentido al conjunto de la ciudad, garantizar trayectos y elementos de continuidad... y ordenar las relaciones entre edificios, equipamientos, monumentos, solares, vías, espacios de transición y espacios abiertos en cada área de la ciudad." (Borja \& Muxí, 2003, pág. 81) 
Pensar en este problema nos llevó a la idea de analizar todo un esquema de trascendencia cultural y social. El espacio público ya no desde su materialización histórica, si no desde la observación contemporánea de la forma en que el espacio público tradicional se ha convertido en una serie de instalaciones para el consumo, los malls o centros comerciales, que incluyen una gama comunicativa visual y sonora, en donde se recurre con exuberancia, a un nuevo lenguaje socio-aspiracional. Sitios en donde la retórica de la forma y la apariencia como signo actualizado de la estética, funden en su propia realidad, el de la obsolescencia de la historia propia, que reconfigura en su contenido, los componentes que dominan la percepción de la realidad del mundo que nos rodea y en el que actuamos. El mundo sensible, así, visible, representa y expresa la experiencia estética que se despliega y acude al mundo de la estructuración espacial de la ciudad, la creatividad se ejerce no como realización que soporta el hacer de la comunidad si no que ejerce de plataforma del mundo cultural de las clases dominantes. Nuevos escenarios convertidos en sitios y lugares de proximidad temporal en los que se desenvuelven las fragmentadas clases sociales de la sociedad de consumo. Espacios convertidos en una falsificación modeladora, que vive la comunidad en su desplazamiento ocioso por lugares de uso aparentemente común. Estas relaciones se concretan sobre el territorio de la ciudad como una nueva especie de convergencia de complejas vinculaciones de pertenencia y apropiación de los lugares sobre el espacio urbano.

La vieja discusión sobre espacio y lugar, sitio y lugar, reconduce parte de esta reflexión, ya que si la ciudad es el lugar de identidad común, por encima de jerarquías sociales, al mismo tiempo sitio de complejas relaciones y proximidad, la idea del espacio se traduce en un categoría consustancial a ambos como extracto de la cultura material edificada y sus funciones en la ciudad. Habría quizás que regresar a tratar de comprender aquello que argumenta Fuentes Gómez cuando explica que: "Los lugares... son resultado de procesos sociales que afectan de diversas formas sus funciones, el tipo de actores y prácticas. Por tanto la necesidad de estudiarlos como parte de una unidad espacial más amplia; la ciudad. Las características arquitectónicas y ambientales de los lugares, usos y funciones, localización en determinada zona de la ciudad, tipo de actores que lo utilizan, etcétera, les confieren papeles y funciones particulares. Éstas pueden ir cambiando por transformaciones en otras partes del espacio urbano... por eso es importante la necesidad de estudiar 'los lugares' sin olvidar la escala espacial más amplia: la ciudad, vista como estructura urbana." (Fuentes Gomez, 2005, pág. 38) En otras épocas, el espacio público, plazas, parques, paseos, alamedas, boulevares, parques urbanos, etc., representaban una de las características esenciales de la ciudad; todas las funciones de la misma las podíamos encontrar en estos espacios, allí se construían las relaciones de las fuerzas sociales, se hacía palpable la vida y el sentido de genius loci (De las Rivas, 1992) de pertenencia y de representatividad; ahora, en la ciudad contemporánea, asistimos a lo que Baudrillard ha definido como la desaparición de la escena y del espacio representativo. ${ }^{3}$ (Baudrillard, 1978) Sólo el simulacro, según los escritos del mismo autor, aparece como substituto de la antigua escena, en este nuevo orden en el que el sentido ya no proviene del referente real, sino de una abstracta construcción de signos, una manipulación de los textos de la arquitectura.

\footnotetext{
${ }^{3}$ En el capítulo de este libro titulado La Precesión de los Simulacros, Baudrillard expone una estimulante interpretación de los fenómenos de la civilización contemporánea como una definitiva «suplantación de lo real por sus signos", en la que «el aspecto imaginario de la representación es barrido por la simulación».
}

ACEO AÑO 12, núm.36, FEBRERO 2018 | EL ESPACIO PÚBLICO CONTEMPORÁNEO EN LA CIUDAD DEL SIGLO XXI, ¿CRISIS O 133 TRASFORMACIÓN? 


\section{El espacio como supuesto}

El crecimiento de la ciudad en el siglo XXI, el desarrollo de los intercambios, el comercio y las comunicaciones, han propiciado la transformación de las actividades tradicionales en él y del espacio público, sobre el que antes se podrían realizar travesías y movilidades desde y en trayectos cortos y de forma peatonal. La ciudad así, es un imaginario construido desde perspectivas diversas que en conjunto, componen una descripción y un relato a través de sus lenguajes y creaciones. Es en los imaginarios que se desatan como proceso social, en donde la ciudadanía y su realización como tal, tienen efecto para la posibilidad de la formación de la ciudad.

El tejido urbano no continuo de la ciudad contemporánea, hace que este mismo se convierta a la vez en una especie de impedimento/barrera para garantizar la conexión entre los espacios públicos. Esta especie de vértebra que conformaban las grandes vialidades y espacios públicos de Roma y París renacentistas y del siglo XIX, o en Guadalajara, a través de vialidades tan importantes como Ave Vallarta y 16 de septiembre, y sus conexiones con la cruz de plazas, Plaza San José, Parque Morelos, Revolución, etc., se conforman en verdaderas conexiones en las que se muestra la extensión pública de la ciudad, hace que la disposición actual de las ciudades a su fragmentación y especialización funcional solamente tomen forma a través de la movilidad y de la adecuación del espacio a las condiciones y requerimientos del automóvil.

Es innegable el grado de simbolismo, representatividad y pertenencia que han distinguido a los espacios públicos a lo largo de la historia. Sus funciones, sus características, su papel en la estructuración de las ciudades; ha sido muy significativa a lo largo de la historia. Los procesos sociales se han representado también allí como parte del devenir de la humanidad, una suma de acciones que se han desarrollado en los espacios colectivos de las ciudades, han caracterizado al mismo tiempo sus cualidades.

A través del tiempo, el intercambio comercial en las ciudades, de realizarse en sus orígenes principalmente a nivel de calle, en los puestos temporales -entre nosotros conocidos como tianguis- y que luego se realizó en pequeñas tiendas en los portales de la ciudad, más tarde en almacenes especializados y posteriormente en las tiendas departamentales, a lo que se anexó como signo de modernidad los llamados supermercados; se realiza ahora principalmente en contenedores cerrados; de ser posible cada vez más grandes y situados principalmente lejos del centro de la ciudad, o en espacios estratégicos de la misma, siempre conectados principalmente por grandes vialidades, preferencialmente cercanos a grandes zonas residenciales de alta plusvalía.

La huella de su transformación, asumida su connotación de espacio de lo público fue adquiriendo a su vez los contenidos del cambio, sobre todo si se entienden estos cambios entre la dimensión de los que se le impusieron a la ciudad y sus funciones. Pensar que ya en su origen, "Cuando los centros comerciales se establecieron dentro de la ciudad, se encerraron en sí mismos, dejando de formar parte de la escena pública." (Gehl \& Gemzoe, 2000, pág. 13) Sin embargo, al formar parte de la trama urbana, su sentido de relación entre lo socialcomunitario y su comprensión de "lugar" se subsume en otras ponderaciones sobre su rol urbano. En esta propuesta, aparecen los gérmenes del asumir que en este proceso, el 
mercado-consumo en su trayectoria y su consecuente "vida en la calle", se ha convertido en algo controlado, que infieren las actividades e interacciones humanas reguladas y envueltas en el discurso de la seguridad.

Es necesario recordar, que el siglo XX ha supuesto, además, un cambio decisivo en las condiciones de uso de la ciudad como lugar de reunión e intercambio de información" (Gehl \& Gemzoe, 2000, pág. 13), sujeta a una serie, ardua y caótica, de graduaciones del orden neoliberal. Sistema que se rehace y requiere otros discernimientos de lo que es público, por parte de los sectores privados dominantes. Paso a paso, el mercado-consumo contemporáneo, ya no es lo mismo que en tiempos pasados, desapareció literalmente de la escena pública para trasladarse a los lugares prefabricados de la esfera privada, para reducir y conducir el contenido de lo público.

Los Centros o Plazas comerciales, malls, suplantan la función histórica de la plaza y la calle tradicional para transformarse en lugares que representan la utopía y aspiraciones de las clases medias y altas; como también de una parte de los sectores menos favorecidos en su proyecto de ascenso a los esquemas de vida más altos de la escala social y se constituyen como islas de funciones particulares en el contexto de la ciudad; significado inequívoco de la fragmentación, dispersión y especialización de la ciudad contemporánea. Los espacios públicos y la estructura total de las urbes, los códigos formales y funcionales de su identidad se ven implicados nuclearmente en el proceso de transformación modernizadora de la ciudad contemporánea, fenómeno que permite su realización bajo la influencia de los escenarios en construcción del siglo XXI.

En los centros comerciales se revalorizan la arquitectura monumental estandarizada, ya que utiliza los mismos signos de referencia en cualquier parte del mundo, además de contener generalmente las mismas tiendas, algunas con formato comercial de franquicia. Esta especie de espacio que adquiere una forma de isla dentro del mar de la ciudad, aquí nos podríamos aventurar a clasificarlo como una producción de "piezas autistas" dentro de la estructura urbana, siguiendo la ruta conceptual de Solá Morales, cuando denomina así a los "fragmentos homogeneizados de la ciudad" que forman parte de las características de la ciudad moderna o contemporánea, refiriéndose que al momento de redactar un plan para ordenar la idea y producción material y funcional de lo urbano. El Plan quedo reducido a un documento "simplísimo y elemental" de carácter exclusivamente jurídico, incorporando sólo "programas orientativos y directrices de sentido común". Lo demás sería el campo de los proyectos físicos (de barrios, centros, espacios públicos), del 'urbanismo para arquitectos' como única responsabilidad de las escuelas de Arquitectura. Sin entender que sin instrumentos potentes de ordenación territorial (metropolitana, comarcal, municipal), estamos en el terreno de las piezas autistas, de los fragmentos homogenizados, destinados a nichos de mercado específicos y a rutinas de marketing demasiado previsibles. (Moya, 2007, pág. 179)

Esta connotación de "autista" deviene de la no-relación implícita que se significa en el marco y contexto urbano, este tipo de espacios, convertidos en un segmento urbano privado-publico, sin una especie de imaginario manifiesto que vaya más allá de sus funciones de consumomercado. El espacio público en su imaginario y realización obliga a pensarlo como el espacio 
recurso, como producto común y como práctica imaginario-estético-simbólico, como entidad social y política de los individuos en comunidad.

La plaza o centro comercial como espacio de acceso público, pero de propiedad y gestión privadas, constituye en la actualidad lo que en el pasado representaban a los espacios públicos centrales de las ciudades. El mall personifica un modelo de desarrollo en forma aislada, que siempre se relaciona con un lugar que tiene límites claros y precisos, que no tienen relación directa con el entorno y en este caso con la trama urbana contigua; su desarrollo es independiente de la estructura urbana y se representa simbólicamente como la utopía del lugar perfecto, las publicidades lo manifiestan claramente, "Plaza del Sol, todo", "Amo compartir, amo disfrutar, amo Guadalajara, amo Andares."

Podríamos debatir con apuntada crítica fenomenológica, que representan una especie de lugar de culto, en su imaginaria analogía con las iglesias. Arquitectura vanguardista y monumental, seguridad que se vende, gran iluminación, en la que formaliza el estilo de su interioridad en donde ser resaltan las ambigüedades ideológicas siempre en pugna. Estos espacios-lugares de la sociedad moderna, que se construyen para ser vistos y disfrutados desde adentro (por fuera nos parecen todos iguales), establecen un modelo de percepción de la realidad exterior y nos muestran en cierta medida aquello de lo que carecemos y hace que las ideas y consumo que incluyen lo visual como anhelo- llenen ese vacío como promesa de un futuro mejor. La analogía entre iglesia y centro comercial se puede ver en el mismo diseño de los espacios; la presencia de la luz en aquellas plazas abiertas o con grandes techos de vidrio, las dobles alturas, el estar allí sin estar con los demás, la relatividad de la cercanía, la presencia de vegetación (posibilidad de crecer, de trascender), todo ello hace que nos aventuremos a considerarlo como espacio de culto. (Klodawsky, 2008)

"El establecimiento de los centros comerciales y la cultura del consumo ha significado un cambio de hábitos en la población, ya que ha llegado a sustituir espacios tradicionales para las actividades cotidianas, ha modificado la vida cultural y la percepción social de la población, ha creado nuevos valores, metas, formas de relacionarse, patrones de consumo y sistemas de referencia." (López Levi, 1999, pág. 23)

La apropiación y utilización particular del espacio (tanto a nivel material como simbólico) así como la transformación de los espacios existentes y la producción de nuevas espacialidades, antes inéditas, en correspondencia con distintos proyectos culturales "emergentes" y en pugna; se encuentran en el vértice de la coartada y realización de lo urbano y de sus habitantes que mueven y concitan el estudio de las ciudades hacia el siglo XXI. "Esta tipología arquitectónica responde a estilos importados, con poca o nula relación con el entorno inmediato, carece de identidad, representa verdaderos refugios de clase que proporcionan la seguridad y el confort que los espacios tradicionales ya no aportan (estacionamiento, vigilancia, climatización, variedad de la oferta, actividades de ocio y esparcimiento, etc.)" (Díaz y Pérez, 2010, pág. 75)

Es aquí donde nos surge la pregunta: ¿Qué se entiende por centros comerciales?, son espacios públicos, son espacios semi públicos, ¿son espacios públicos de gestión privada? 0

${ }^{4}$ Algunas de estas son: <www.plazadelsol.com>, <www.andares.com> Fecha de consulta: 04 junio 2015 
se podrán considerar como espacios privados? Las características, las funciones, el grado de conexión o no con la ciudad etc., de los centros comerciales, plazas comerciales, malls, así como el estilo de vida que establecen, su conversión en modelos para el ocio y el consumo, han sido estudiadas por diferentes autores principalmente analizando sus características de uso y apropiación.

Coincidimos con el concepto referido por Fuentes Gómez cuando cita a López Levi: "quien define el centro comercial compuesto por un grupo de negocios que se ubican en uno o más niveles, localizados entre grandes almacenes por tiendas departamentales que sirven de polo de atracción. Esta estructura garantiza un flujo estable de compradores y paseantes. Sin embargo, es un lugar que va más allá de lo concreto. Es un conjunto arquitectónico que rebasa las fronteras de la realidad e incorpora elementos provenientes de la imaginación y los deseos. Es un paisaje organizado para promover la fantasía y el placer. Su ambiente está influido por las imágenes de la televisión. La arquitectura resulta monumental, compleja y sus acabados son más lujosos que los de sus predecesores, los antiguos centros comerciales que consistían en una hilera de tiendas frente a un estacionamiento. (Op. cit, 21-22, citado por Fuentes Gómez p.p. 250-251.)

Podemos establecer la definición de la plaza o centro comercial como una edificación de uso comercial compuesto por negocios que ofrecen diferentes tipos de productos. Está conformado por una o dos grandes tiendas departamentales que se establecen en los extremos del espacio, llamadas tiendas anclas, ya que sirven de polo de atracción a los demás comercios establecidos. Pueden tener uno o varios niveles, cuentan con estacionamiento, áreas de juegos infantiles, áreas de comida, salas de cine, casinos, bancos, y ofrecen diferentes tipos de servicios y establecimientos para el ocio y el consumo, cafés, restaurantes y espacios para realizar pequeños espectáculos, conciertos y festivales. Es un lugar al que se va, con entrada y salida (barreras disuasorias validas). Este complejo arquitectónico está pensado para que el usuario permanezca mucho tiempo realizando sus compras o actividades de ocio, por ello se idearon que fueran techadas, para poder tener la posibilidad de que las tiendas estuvieran abiertas todo el tiempo. (Klodawsky, 2008)

"En términos comerciales, el fenómeno del encierro urbano tiene su máxima expresión en las plazas comerciales, que podrían definirse como un subcentro urbano virtual conformado por un grupo de locales comerciales reunidos en uno o varios edificios asociados, planeados y administrados como una ciudad operativa, aislados del entorno urbano que los rodea, con un ambiente hiperreal construido a partir de un escenario destinado a la manipulación de los sentidos y la razón para lograr un mayor consumo por parte de los visitantes y mayor ganancia para los dueños del lugar." (López Levi, 2006, págs. 156-157)

Como un punto relacionado, que aquí no es posible extender, pero que quisiera dejar implícito como una preocupación, se agrega la conectividad por los medios electrónicos de los ciudadanos, que no obstante en una gran mayoría no tiene acceso a las nuevas TIC, y a la información que distribuyen y sugieren imaginarios en el espacio-lugar-de lo público. No olvidemos la proximidad renovada del proyecto de ciudad-espacio público virtual para la reproducción del modelo de vida y el sistema político económico de hacer ciudad, que corresponde a las ambiciones del poder ligado a los dominantes mediático-ideológicas en el mundo globalizado de la acumulación y la dependencia de las regiones periféricas. 
El espacio público, recordando a Habermas, se encuentra además en: “... el grueso de las noticias, películas, programas de televisión, videos, libros, discos, imágenes fotográficas, ideas, movimientos artísticos que circulan por el mundo proviene de unos pocos centros de producción cultural global. Una veintena de corporaciones (en su mayoría estadounidenses) se reparten la parte del león de la cultura de masas global: Disney, Time-Warner-Turner-AOL, Viacom, TCI, ATT, Westinghouse, General Electric, DreamWorks, IBM, Microsoft, News Corporation (Australia), Sony y Matsushita (Japón), Bertelsmann (Alemania), Pearson (Gran Bretaña), Philips (Holanda), Seagram-MCA-Universal (Canadá), Televisa (México), TV Globo (Brasil). Unos pocos gerentes generales -Michael Eisner, Sumner Redstone, John Malone, Laurence Tisch, Ted Turner, Michael Jordan, Gerald Levin, Robert Wright, Steven Spielberg, Bill Gates, Rupert Murdoch- inciden en forma desproporcionada en cómo la población mundial piensa, siente, imagina, sueña y participa de la civilización actual, virtualizando como real toda idea de una esfera pública democrática global." (Remedi, 2004, pág. 10)

\section{Conclusiones}

El papel de los centros y plazas comerciales como espacios privado-públicos de la ciudad contemporánea, por su apropiación, funciones y significados, son espacios representativos del consumo, el ocio y el esparcimiento de la vida moderna. Así mismo, la función de estos lugares-espacios-edificios en la ciudad, se conforman como partes independientes, aisladas y sin relación con el contexto de lo urbano total, mientras al mismo tiempo se sirven de la estructura funcional de la ciudad socialmente edificada, convertidos en especie de islas con acceso restringido, espacios encerrados en sí mismos, que han hecho que el comercio no forme parte de la escena pública como proximidad y parte del imaginario abierto en su historia. En resumen, estructuras arquitectónicas diseñadas no para ser vistas y admiradas desde afuera, sino disfrutadas interiormente; espacios de exclusividad para personas identificadas no como ciudadanos sino como consumidores.

Estos espacios públicos se convierten en una especie de nodos autónomos; si se analiza su vinculación con la estructura urbana; si observamos sus usos y significados, se convierten en espacios independientes de la vida ciudadana que contribuyen a la dispersión y a la concentración individualizada que confirma las características de: fragmentación/dispersión de la ciudad contemporánea.

Es por lo tanto interesante recordar algo que escribió Fernando Roch hace algunos años, acerca de: "...el papel fundamental que tiene el aparato comercial en la construcción, transformación, mantenimiento, vitalización y también destrucción de la ciudad, y no es aventurado decir que sea cual sea la ciudad del futuro tendrá que resultar de una convivencia articulada de todas las formas comerciales que han surgido a lo largo de su historia." (Roch Peña, 1991, pág. 26)

De hecho, el auge que ha tenido en los últimos años la venta online, entre otros motivos, ha resultado que tiendas reconocidas cambien la estrategia de ubicación, abandonan la periferia y apuestan por retomar la zona comercial tradicional del centro de las ciudades, revitalizando y 
reforzando los beneficios de la centralidad para poder seguir manteniéndose en el mercado (Mango, Inditex, Media Markt en España) (Salvatierra, 2017).

En esto ha influido además, el auge de los llamados millenials, jóvenes que apuestan más por las experiencias y los viajes que por la compra de bienes como ropa. En el año 2017 se clausuraron en EU más de 3, 500 tiendas que se ubicaban en centros comerciales o malls, evidenciado con ello que el modelo también está pasando por un período de crisis principalmente el países desarrollados.

\section{Bibliografía}

BORJA, J y MUXI, Z. El espacio público: ciudad y ciudadanía. Barcelona, Electra, 2003, 415 p.

DÍAZ, V. y PÉREZ, M. T. Ciudad, espacio público y construcción ciudadana. En: ACE: Architecture, City and Environment [en línea] 25 Octubre 2010, vol. 5, núm. 13, p. 67-78. [Fecha de consulta: 14 agosto 2017]. Disponible en: <http://hdl.handle.net/2099/9345> DOI: <http://dx.doi.org/10.5821/ace.v5i14.2506>

FUENTES GÓMEZ, J. H. Espacios, lugares, actores, prácticas e imaginarios urbanos en Mérida, Yucatán, México. Mérida, México, Ediciones de la Universidad Autónoma de Yucatán, 2005, $389 \mathrm{p}$.

BAUDRILLARD, J. Cultura y simulacro. Barcelona, Editorial Kaidós, 1978, 185 p.

DE LAS RIVAS, J. L. El espacio como lugar, sobre la naturaleza de la forma urbana. Serie de Arquitectura y Urbanismo № 19, Servicio de Publicaciones Universidad de Valladolid, 1992, $216 \mathrm{p}$.

GEHL, J. y GEMZOE, L. Nuevos espacios urbanos. Barcelona, Gustavo Gili, 2000, 261 p.

KLODAWSKY, H. Los templos del consumo [en línea] 2008 [Fecha de consulta: 20 Julio 2017] Disponible en: <https://www.youtube.com/watch?v=HUaFapTIMp8>

LÓPEZ LEVI, L. Centros comerciales: espacios que navegan entre la realidad y la ficción [en linea] 1999 [Fecha de consulta: 14 agosto 2017] Disponible en:

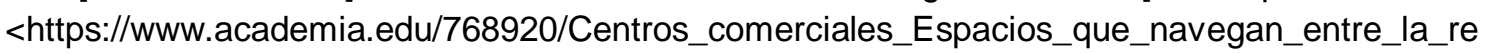
alidad_y_la_ficción>

MOYA, L. Vivienda reducida [en línea] Universidad Politécnica de Madrid, UPM, Mairea libros, Madrid, 2007, 215 pp. [Fecha de consulta: 24 agosto 2014] Disponible en: $<$ http://polired.upm.es/index.php/urban/article/viewFile/494/496>

REMEDI, G. La ciudad latinoamericana SA (o el asalto al espacio público) En: Las dimensiones del espacio público. Problemas y proyectos, Buenos Aires, Gobierno de la Ciudad de Buenos Aires / Subsecretaría de Planeamiento [en línea] 2004, pp. 7-8. Disponible en: <http://www.henciclopedia.org.uy/autores/Remedi/CiudadLatinoamSA.htm> 
ROCH PEÑA, F. Reflexiones sobre el espacio público comercial, fin de siglo En: Revista Urbanismo: revista oficial del Colegio de Arquitectos de Madrid [en línea] ISSN 0213-9391, oㅡ 14, 1991, págs. 20-27 [Fecha de consulta: Agosto 2017] Disponible en: <https://www.coam.org/media/Default\%20Files/fundacion/biblioteca/revista-urbanismo/docs/re vista-urbanismo-n14-pag20-27.pdf>

SALVATIERRA, J. Zara abre en Madrid su tienda más grande del mundo En: El País, Economía [en línea] 2017 [fecha de consulta: 7 abril 2017] Disponible en <https://economia.elpais.com/economia/2017/04/06/actualidad/1491489624_834856.html> 